\title{
Rapidly Tunable Microring Filters for Optical Add-Drop (Project Report CIAN2-1-Y5)
}

\section{SHAYAN MOOKHERJEA}

Department of Electrical and Computer Engineering, University of California, San Diego, MC 0407 La Jolla CA 92093-0407 USA

Email:smookher@ucsd.edu

\section{Introduction and Goals}

This was a project under the Thrust 2 "Subsystem Integration and Silicon Nanophotonics" of the NSF-funded Center for Integrated Access Networks. The goal of this research was to design, fabricate and test microchip-scale silicon photonic components for optical WDM (wavelength division multiplexed) add/drop functionality in access and data networks. Designs were submitted for manufacturing in 2014 and 2016, and diced microchips were returned to the designers for testing after about 1 year following design submission.

Figure 1 shows an image of the test site, a microchip die-attached to a carrier printed circuit board with wirebonded electrical lines, and an array of fibers used to interrogate grating couplers on the chip for optical input/output.
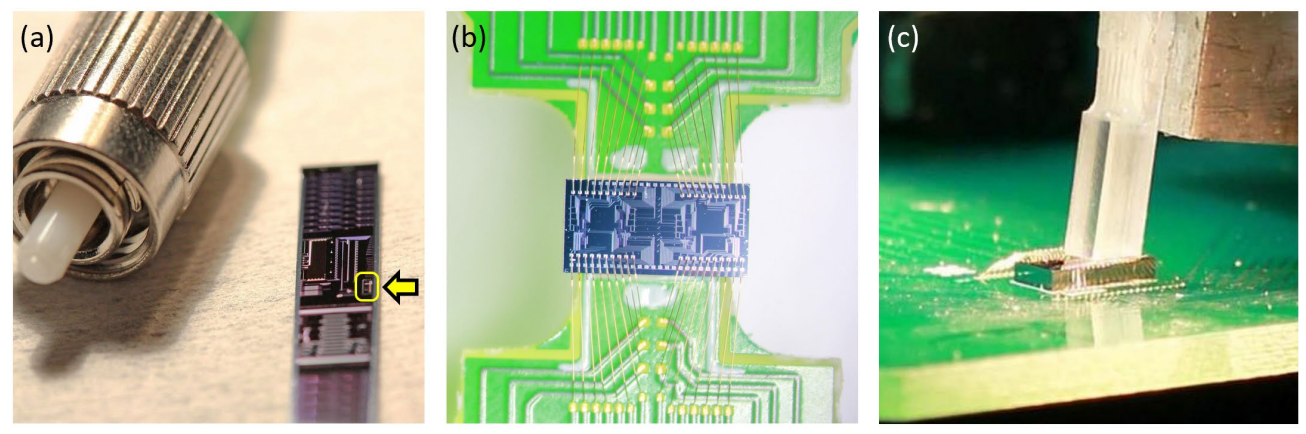

\footnotetext{
Figure 1 (a) Image of a multi-project silicon photonic chip, with the relevant section marked using a yellow rectangle, which provides add/drop functionality and variable optical attenuation (VOA) functionality for up to four WDM channels. (b) Chip mounted on PCB and wirebonded electrical driver connections (microheaters). (c) Optical IO using an array of high-NA fibers addressing grating couplers on the chip.
}

The purpose of this chip is as follows: a group of four C-band WDM channels (externally modulated with $10 \mathrm{Gbit} / \mathrm{s} \mathrm{NRZ}$ data from commercial transceivers at fixed wavelengths) could be added or dropped from a bus waveguide which contains up to 24 WDM channels. The add-drop functionality was provided using coupled-microring filters. The channels were tunable over the C-band using thermal tuning of the microrings. Here, the goal was to achieve rapid tunability at least in one direction. We were able to tune across 26 nanometers in about 0.6 microseconds. This was achieved by using a pre-emphasized 
electrical driving waveform for the heaters, and designing the microring resonators to withstand such waveforms without damage.

This chip was intended for use in a campus ring network (UCSD "MORDIA") using for networking research. This chip was designed to achieve the same functionality as was previously achieved on each node of the testbed using an assembly of conventional off-the-shelf optical components. Off-chip lasers were used for testing. A limited amount of packaging was performed to assist in testing as shown in Figure. 1.

\section{Activities}

Previously we reported on an earlier version of the test microchip [1] tested on a 24-channel wavelength division multiplexed (WDM) optical network testbed "MORDIA" at UC San Diego [2]. The wavelength usage plan consisted of six groups of four WDM channels, each modulated at $10 \mathrm{~Gb} / \mathrm{s}$ using the NRZ format. The wavelength usage plan was not exactly periodic, as some channels were left unused. Thus, tunability across the entire $\mathrm{C}$-band was required of the add and drop components. The system requirement was to drop the four channels in one group, and add channels individually.

(a)

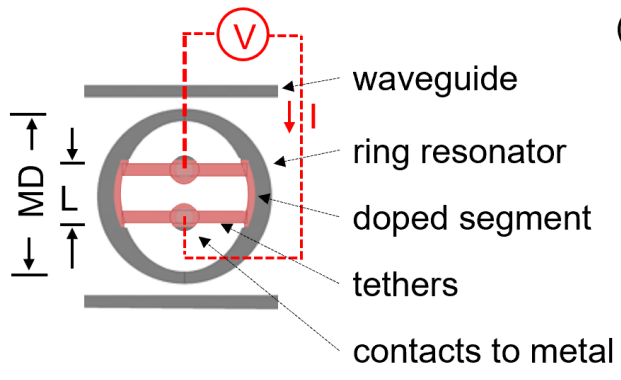

(b)

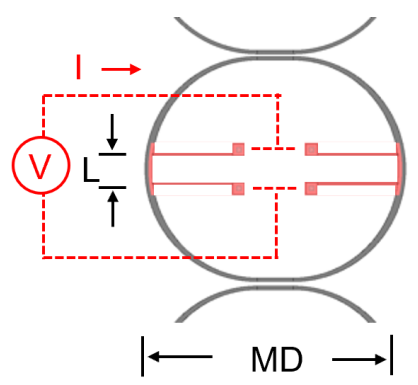

(c)

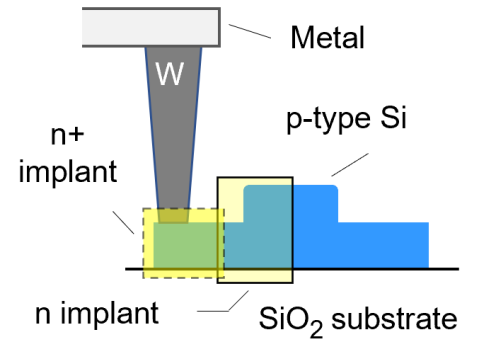

(d)

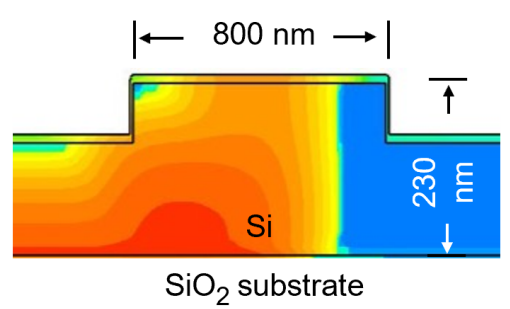

Net doping $\left(10^{\mathrm{x}} / \mathrm{cm}^{3}\right)$

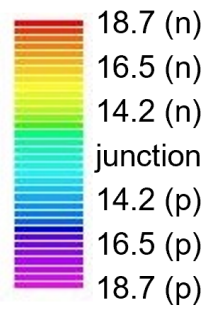

Figure 2 Design of the thermally-tunable silicon microrings used in the drop and add filter stages. Dopants are incorporated within the microrings so that electrical heating is efficiently translated into an optical index change.

Figure 2 shows the design principles (not an actual layout) of the thermallytuned silicon microring resonators used in the filters. Panel (a) shows a smallradius ring with a free spectral range (FSR) greater than $30 \mathrm{~nm}$. These rings 
were used in the drop section of the overall design. A larger ring with an FSR of about $800 \mathrm{GHz}$ was used in the add section, which followed the drop section in the overall chip layout (see Year 3 project report).

An innovative design was used in the waveguide cross-section. The silicon which comprised the waveguide core was lightly p-doped. A portion of the waveguide cross-section as shown in Fig. 2, panel (c) was doped with $n$ implants. When an electrical current was passed through this section, Joule heating caused a temperature rise in the silicon, altering its refractive index. The thermo-optic effect in silicon is significant, and can shift the resonance wavelength by tens of nanometers for the small rings.

A nanoscale heater that occupies a portion of an optical waveguide has to be designed carefully. Measurements of the first batch of devices showed they were susceptible to premature failure as the driving voltage was increased. An improved design proved to be more robust by taking advantage of an interesting physics phenomenon. A narrow current transport channel consisting of a small n-doped region surrounded by a p-doped region (and the etched walls of the silicon waveguide) is pinched off at higher voltages, and thus the current is limited as the voltage increases, as shown in Figure 3(a). Since voltage continues to increase, the heater dissipates more power (product of voltage and current) and causes a correspondingly larger thermo-optic index shift. However, the clamped current prevents electro-migration and damage to the device which is usually seen to occur quite easily in a conventional resistive heater of such small dimensions.

The filters used single or coupled silicon microrings with directional couplers. In this project, we had experimentally studied the dispersion of directional couplers using a microring coupled to a waveguide [3]. An accurate knowledge of dispersion is also necessary for a wide range of integrated photonics devices, such as coupled-microring filters [4], some of which are included in the layout shown in Figure 1 (also see Fig. 6). At high resonant optical powers, silicon microring resonators can exhibit nonlinear behavior, such as bistability [5]. However, these effects are not expected to be manifest at the power levels used in this project.

\section{Progress and Impact}

A benefit of the current-limiting effect of the integrated nanoheaters is that preemphasis "spikes" can be added to the heater driving waveform, in order to speed up the effect. This is shown in the experimental measurements reported in Fig. 3(b). Pre-emphasis is a technique that is often used with p-n junction modulators to overcome the low-pass filter roll-off characteristics and achieve a faster tuning speed. Here, the same concept was applied to thermo-optic heaters, which also present a low-pass roll-off characteristic. 

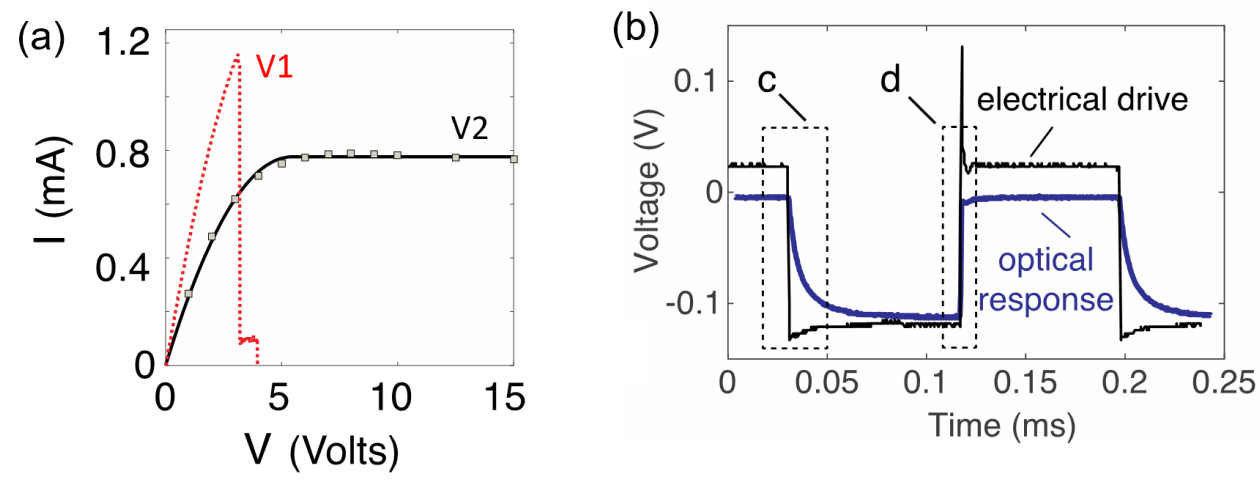

Figure 3(a) I-V characteristics of the heaters integrated within the silicon microring resonators, as shown in Figure 2. (b) The V2 design allowed for pre-emphasis in the electrical drive waveform (feature $\mathrm{d}$ ) which resulted in a much faster response, with a time-constant that was more than 20 times faster than that of the "natural" ring-down response of the system (feature c).

Figure 4 shows the time constants for the cooling response of the system and the forced heating response, with a pre-emphasis electrical driving waveform. The latter is much faster, and could be used to tune the microring resonator across $25 \mathrm{~nm}$ wavelength span in less than 0.6 microseconds.

(a)

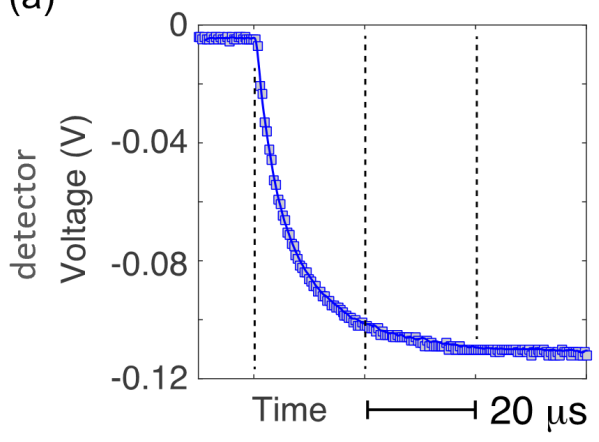

(b)

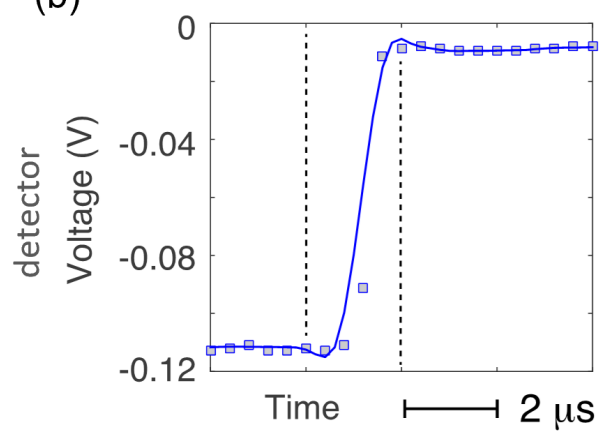

Figure 4 (a) When the voltage to the integrated heater is dropped to zero, the optical transmission changes with a characteristic time constant as the heat is dissipated. The fall time is about 20 microseconds. (b) The rise time with a pre-emphasis waveform is much shorter, and is less than 1 microsecond.

Coupled microrings were used to achieve a more versatile and tunable filtering response compared to single microring resonators. They can achieve a flat-top transmission passband with steeper sidewalls, as shown in Figure 5. These measurements were performed by tuning one resonator in a coupled-resonator system slightly away from the other. Previously, we have developed the theory and design principles of coupled microring resonators, also known as Coupled Resonator Optical Waveguides (CROWs). A tight-binding model of bandcenter propagation was formulated [6], and theoretical studies of nonlinear propagation were developed on this basis [7]. Studies were performed to 
examine the regions of high dispersion which may be relevant to signal distortion when filtering wideband signals [8] or all-optically tunable dispersion compensation devices [9]. To help in designing structures in silicon photonics, we have developed an extension of coupled-mode theory which is applicable to directional couplers in high index contrast materials, in coupledwaveguide structures [10] and coupled-resonator structures [11].

(a)

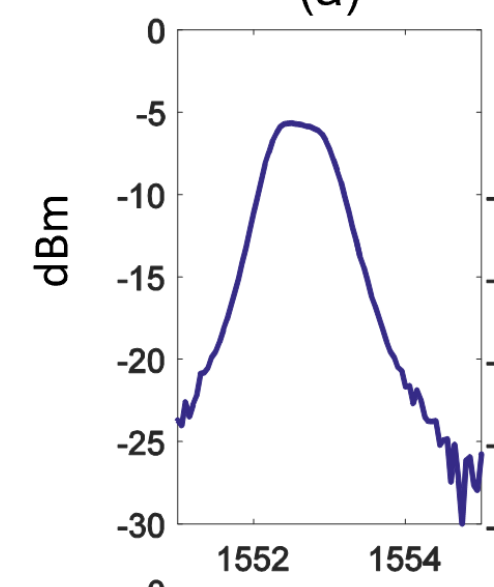

(b)

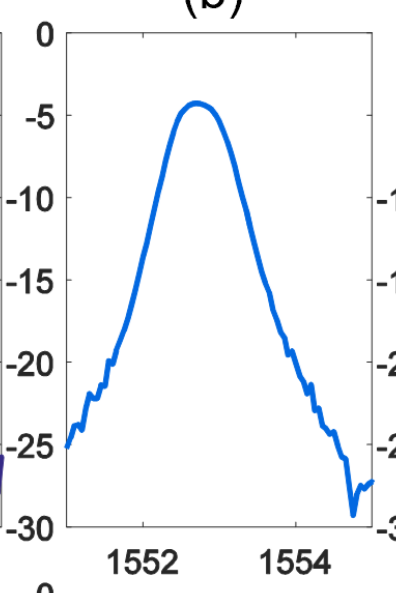

(c)

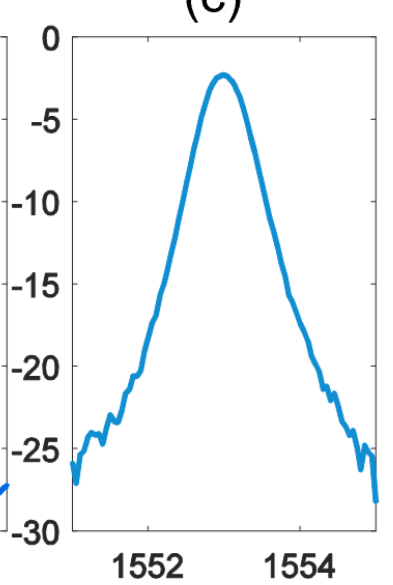

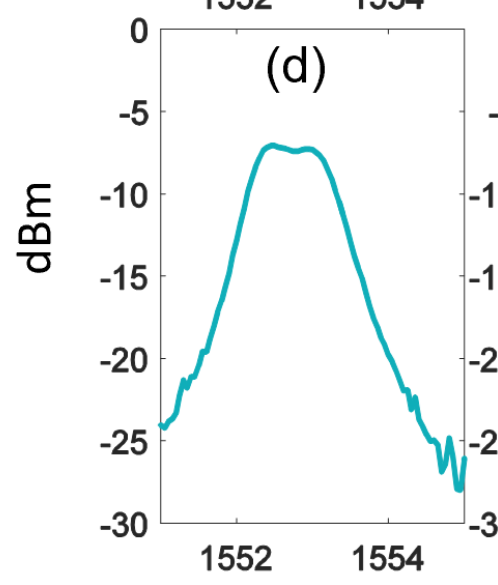
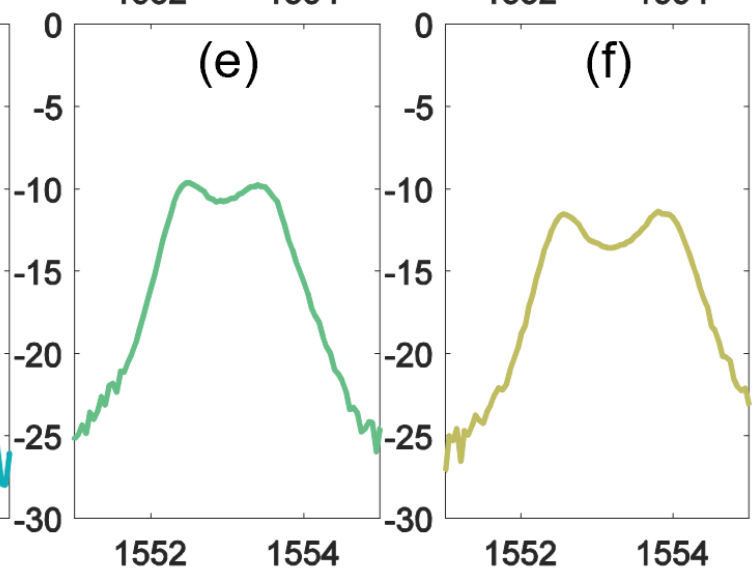

Wavelength $(\mathrm{nm})$

Figure 5 Panels (a) through (f) show the measurements of a coupled-microring resonator filter as one resonator is thermally shifted with respect to the other. Panel (c) shows the case when the resonances are aligned most closely and has the highest overall transmission and narrowest bandwidth.

A collaboration with IBM's silicon photonics group (Dr. W. M. J. Green) has provided insights into silicon photonics fabrication and also supported technical collaboration on fabricating structures at wafer-scale using silicon photonics processing [12], [13]. Dr. Ivan B. Divliansky (CREOL, Florida) collaborated with us for the electron-beam lithography of long waveguide structures [14]. CROWs of up to 235 coupled resonators were successfully fabricated and 
measured, and a good agreement was achieved between experiments and theory [13], [15], [16].

Impact on Human Resources: One graduate student in the PI's group worked on this topic as part of their education and training. The Center provided extensive opportunities to interact with researchers and students from other groups and universities as well as with researchers from Sandia.

\section{Subsequent Extensions}

Driving an electrical current through a forward biased p-n silicon diode causes heating. Heaters can be formed in doped silicon regions $(50 \mu \mathrm{m}$ long, $1 \mu \mathrm{m}$ wide) placed about $0.7 \mu \mathrm{m}$ away from optical waveguides in which the light is well confined. Using multiple diodes, about $21 \mathrm{~mW}$ of dissipated power was used to achieve pi phase shift in a Mach-Zehnder interferometer structure [17].

CROW structures can have other applications in photonics, such as the generation of entangled photon pairs [18], and developing novel amplifiers and lasers [19]. For example, the resonator-enhanced nonlinearity benefits the generation of entangled photon-pair and heralded single-photon generation using silicon photonics [20]. Our initial measurements of spontaneous fourwave mixing (SFWM) were on correlated photon-pair generation and heralded single-photon generation. An experimental measurement of entanglement was performed later, using a two-photon interferometer constructed to verify timeenergy entanglement [20]. Structures such as VOA's can be useful in improving the amplitude balance between different optical pathways on a microchip circuit. VOA's in silicon photonics can be tuned rapidly (at sub nanosecond speeds) are also useful to protect sensitive detectors from unwanted levels of input optical power, such as the so-called "blinding attacks" on single-photon avalanche diodes.

These microring filters are tuned using the thermo-optic effect. Although the tuning speed was greatly improved from milliseconds to microseconds, there are some applications that require even faster tunability. For such applications, electronic controllability can be of interest, especially in the coupled-microring structure [21]. Carrier-driven effects can achieve sub-nanosecond speeds of varying the optical index and absorption. Even faster effects can be achieved using ferro-electric materials which decouple the amplitude and phase effects. For example, optically-transparent thin-film ferro-electric materials bonded to silicon waveguides can be used to achieve phase shifters with more than 100 $\mathrm{GHz}$ of bandwidth, as was demonstrated later [22].

Microring resonators are sensitive to disorder. An example of the impact of disorder on a coupled-microring resonator filter bank is shown in Fig. 6. As a way to compensate for defects, we studied potential methods for precisely tuning resonators without heaters. Our method is based on field-induced local 
oxidation of $\mathrm{Si}$ to $\mathrm{SiO}_{2}$ via a chemical reaction near an electrically-biased conducting atomic-force microscope tip [23]. A single silicon microresonator can be monitored and controlled more easily [24] than a device that consists of many coupled resonators. Infrared imaging can be used to study light propagation through these structures [14], [25] and is a useful tool for finding defects or damaged sites. The first generation of microrings with inbuilt heaters in particular were quite easily damaged when tuning was attempted over wide spectral ranges and infrared imaging was useful in re-designing the devices to withstand heating over a wider range.

(a)

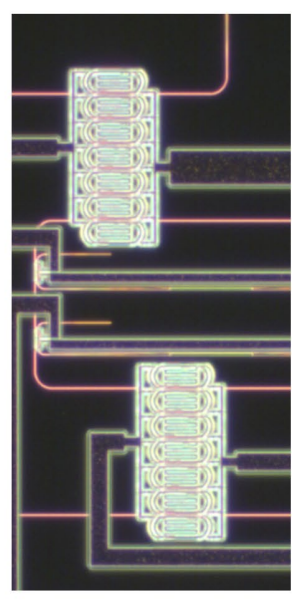

(b)

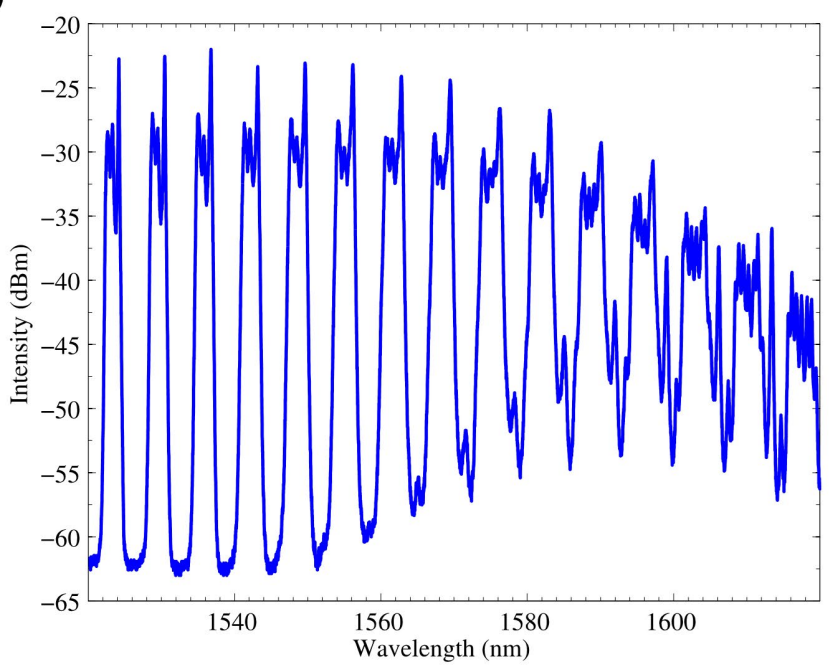

Figure 6 (a) Darkfield optical microscope image of a two-section tunable microring filter, where each section consists of five coupled microring resonators. (b) Due to disorder in the coupling coefficients, the transmission spectrum of the filter bank was degraded, with a high ripple in the passband, and a drop-off in absolute transmission at longer wavelengths, even though the passbands are wider, contradicting the theory.

\section{Open-Access Reporting Initiative}

PRAISE: This open-access document is provided in support of our PRAISE

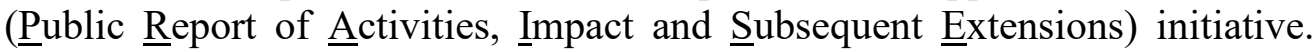
What is it? An open-access document shared with the public which describes the research outcomes of publicly-funded projects. For us, these projects are typically funded by the NSF (National Science Foundation).

\section{References}

[1] R. Aguinaldo et al., "Silicon photonic integrated components for add, drop, and VOA in a 4-channel data-center network," in 2014 IEEE 
Photonics Conference, San Diego, CA, Dec. 2014, pp. 1-2. doi: 10.1109/IPCon.2014.7092976.

[2] R. Aguinaldo et al., "Wideband silicon-photonic thermo-optic switch in a wavelength-division multiplexed ring network," Opt. Express, vol. 22, no. 7, p. 8205, Apr. 2014, doi: 10.1364/OE.22.008205.

[3] R. Aguinaldo, Yiran Shen, and S. Mookherjea, "Large Dispersion of Silicon Directional Couplers Obtained via Wideband Microring Parametric Characterization," IEEE Photon. Technol. Lett., vol. 24, no. 14, pp. 1242-1244, Jul. 2012, doi: 10.1109/LPT.2012.2198639.

[4] J. R. Ong, R. Kumar, and S. Mookherjea, "Silicon microring-based wavelength converter with integrated pump and signal suppression," Opt. Lett., vol. 39, no. 15, p. 4439, Aug. 2014, doi: 10.1364/OL.39.004439.

[5] S. Mookherjea and M. A. Schneider, "The nonlinear microring add-drop filter," Opt. Express, vol. 16, no. 19, p. 15130, Sep. 2008, doi: 10.1364/OE.16.015130.

[6] S. Mookherjea and A. Yariv, "Pulse propagation in a coupled resonator optical waveguide to all orders of dispersion," Phys. Rev. E, vol. 65, no. 5, p. 056601, Apr. 2002, doi: 10.1103/PhysRevE.65.056601.

[7] S. Mookherjea and A. Yariv, "Optical pulse propagation and holographic storage in a coupled-resonator optical waveguide," Phys. Rev. E, vol. 64, no. 6, p. 066602, Nov. 2001, doi: 10.1103/PhysRevE.64.066602.

[8] S. Mookherjea, D. S. Cohen, and A. Yariv, "Nonlinear dispersion in a coupled-resonator optical waveguide," Opt. Lett., vol. 27, no. 11, p. 933, Jun. 2002, doi: 10.1364/OL.27.000933.

[9] S. Mookherjea, "Using gain to tune the dispersion relation of coupledresonator optical waveguides," IEEE Photon. Technol. Lett., vol. 18, no. 5, pp. 715-717, Mar. 2006, doi: 10.1109/LPT.2006.871144.

[10] M. L. Cooper and S. Mookherjea, "Numerically-assisted coupledmode theory for silicon waveguide couplers and arrayed waveguides," Opt. Express, vol. 17, no. 3, p. 1583, Feb. 2009, doi: 10.1364/OE.17.001583.

[11] S. Mookherjea, "Spectral characteristics of coupled resonators," $J$. Opt. Soc. Am. B, vol. 23, no. 6, p. 1137, Jun. 2006, doi: 10.1364/JOSAB.23.001137.

[12] J. R. Ong et al., "Low-power continuous-wave four-wave mixing in silicon coupled-resonator optical waveguides," Opt. Lett., vol. 36, no. 15, pp. 2964-2966, 2011.

[13] M. L. Cooper et al., "235-ring Coupled-Resonator Optical Waveguides," in Conference on Lasers and Electro-Optics 2010, San Jose, California, 2010, p. CTuHH3. doi: 10.1364/CLEO.2010.CTuHH3.

[14] M. L. Cooper, G. Gupta, J. S. Park, M. A. Schneider, I. B. Divliansky, and S. Mookherjea, "Quantitative infrared imaging of silicon-on-insulator 
microring resonators," Opt. Lett., vol. 35, no. 5, p. 784, Mar. 2010, doi: 10.1364/OL.35.000784.

[15] S. Mookherjea and M. A. Schneider, "Avoiding bandwidth collapse in long chains of coupled optical microresonators," Opt. Lett., vol. 36, no. 23, p. 4557, Dec. 2011, doi: 10.1364/OL.36.004557.

[16] M. L. Cooper and S. Mookherjea, "Modeling of Multiband Transmission in Long Silicon Coupled-Resonator Optical Waveguides," IEEE Photon. Technol. Lett., vol. 23, no. 13, pp. 872-874, Jul. 2011, doi: 10.1109/LPT.2011.2141657.

[17] A. Ribeiro and W. Bogaerts, "Thermo-optical phase shifter with integrated diodes for multiplexed control," in Optical Fiber Communication Conference, San Diego, California, 2018, p. Th2A.4. doi: 10.1364/OFC.2018.Th2A.4.

[18] J. R. Ong and S. Mookherjea, "Quantum light generation on a silicon chip using waveguides and resonators," Opt. Express, vol. 21, no. 4, p. 5171, Feb. 2013, doi: 10.1364/OE.21.005171.

[19] S. Mookherjea, "Semiconductor coupled-resonator optical waveguide laser," Appl. Phys. Lett., vol. 84, no. 17, pp. 3265-3267, Apr. 2004, doi: 10.1063/1.1719278.

[20] R. Kumar, M. Savanier, J. R. Ong, and S. Mookherjea, "Entanglement measurement of a coupled silicon microring photon pair source," Opt. Express, vol. 23, no. 15, p. 19318, Jul. 2015, doi: 10.1364/OE.23.019318.

[21] S. Mookherjea, J. R. Ong, X. Luo, and L. Guo-Qiang, "Electronic control of optical Anderson localization modes," Nature Nanotech, vol. 9, no. 5, pp. 365-371, May 2014, doi: 10.1038/nnano.2014.53.

[22] X. Wang, P. O. Weigel, J. Zhao, M. Ruesing, and S. Mookherjea, "Achieving beyond-100-GHz large-signal modulation bandwidth in hybrid silicon photonics Mach Zehnder modulators using thin film lithium niobate," APL Photonics, vol. 4, no. 9, p. 096101, Sep. 2019, doi: 10.1063/1.5115243.

[23] Y. Shen, I. B. Divliansky, D. N. Basov, and S. Mookherjea, "Perfect set-and-forget alignment of silicon photonic resonators and interferometers," in Optical Fiber Communication Conference/National Fiber Optic Engineers Conference 2011, Los Angeles, California, 2011, p. PDPC3. doi: 10.1364/OFC.2011.PDPC3.

[24] M. Savanier, R. Kumar, and S. Mookherjea, "Optimizing photon-pair generation electronically using a $p-i-n$ diode incorporated in a silicon microring resonator," Appl. Phys. Lett., vol. 107, no. 13, p. 131101, Sep. 2015, doi: 10.1063/1.4932047.

[25] S. Mookherjea and H. R. Grant, "High dynamic range microscope infrared imaging of silicon nanophotonic devices," Opt. Lett., vol. 37, no. 22, p. 4705, Nov. 2012, doi: 10.1364/OL.37.004705. 
